\title{
El Doctorado Latinoamericano en Educación: una experiencia de internacionalización y migración académica en la posgraduación
}

\begin{abstract}
The Latin American Doctorate in Education: an Experience of Internationalization and Academic Migration in postgraduate studies
\end{abstract}

\section{O Doutorado Latino-americano em Educação: uma experiência de internacionalização e migração acadêmica na pos-graduação}

Luisa Fernanda Mejía Toro

Doctora en Educación

Universidad Federal de Minas Gerais

profesoraluisamejia@gmail.com

Lívia Maria Fraga Vieira

Doctora en Ciencias de la Educación

Universidad Federal de Minas Gerais

liviafraga59@gmail.com

Juliana de Fátima Souza

Doctora en Educación

Universidad Federal de Minas Gerais

jusouzarp@gmail.com

\section{Resumen}

Este artículo presenta la experiencia de intercambio y migración de estudiantes latinoamericanos y caribeños en el ámbito del Doctorado Latinoamericano en Educación: Políticas Públicas y Profesión Docente (DLA), desarrollado en la Universidad Federal de Minas Gerais. Esta experiencia es resultado de un acuerdo internacional, firmado en el año 2008 entre 11 universidades de 8 países y con la intervención de la UNESCO y la Red Kipus. El curso fue implementado a partir del año 2010 en la UFMG, y ha permitido el encuentro de 61 estudiantes, 29 brasileños y 32 provenientes de otros países 
del Sur. El análisis se basó en un estudio documental y en las respuestas a cuestionarios aplicados a egresados del programa y buscó comprender los sentidos de un posgrado de alto nivel en educación, con una mirada latinoamericana. El DLA ofrece una formación gratuita y de calidad, que da garantías para que sus alumnos puedan desarrollar sus estudios, conectarse con otra lengua y cultura y desde allí aportar elementos nuevos para la investigación en políticas públicas y educación. Los encuentros que propicia este programa han contribuido con la formación de investigadores con una visión regional amplia y crítica de la cuestión educativa, fortaleciendo lazos de cooperación y de trabajo duraderos. Además, esta experiencia introdujo en el programa de posgraduación brasileño la necesidad de establecer relaciones solidas con la producción teórico científica latinoamericana en educación, llevando también la formación de los docentes brasileños a la tarea de orientar tesis desde perspectivas comparada en América Latina.

Palabras clave: Migraciones estudiantiles Sur-Sur, posgraduación, América Latina, Doctorado Latinoamericano en Educación, UFMG.

\section{Summary}

This article presents the experience of exchange and migration of Latin American and Caribbean students in the field of the Latin American Doctorate in Education: Public Policies and Teaching Profession (DLA), developed at the Federal University of Minas Gerais. This experience is the result of an international agreement, signed in 2008 between 11 universities from 8 countries and with the intervention of UNESCO and the Kipus Network. The course was implemented in 2010 at UFMG, and has allowed the meeting of 61 students, 29 Brazilians and 32 from other countries of the South. The analysis was based on a documentary study and on the responses to questionnaires applied to graduates of the program and sought to understand the meanings of a high-level postgraduate degree in education, with a Latin American perspective. The DLA offers free and quality training, which guarantees that its students can develop their studies, connect with another language and culture and from there contribute new elements for research in public policies and education. The meetings fostered by this program have contributed to the training of researchers with a broad and critical regional vision of the educational issue, strengthening long-lasting ties of cooperation and work. In addition, this experience introduced in the Brazilian postgraduate program the need to establish solid relationships with Latin American scientific theoretical production in education, also leading the training of Brazilian teachers to the task of guiding thesis from comparative perspectives in Latin America.

Keywords: South student migration, posrgraduate, Latin America, Latin American Doctorate in Education, UFMG.

\section{Resumo}

Esse artigo apresenta a experiência de intercâmbio e migração de estudantes latino-americanos e caribenhos no âmbito do Doutorado Latino-americano em Educação: Políticas Públicas e Profissão Docente (DLA), desenvolvido na Universidade Federal de Minas Gerais (UFMG). Essa experiência é resultado de um acordo internacional, assinado em 2008 entre 11 universidades de 8 países com a intervenção da UNESCO e a rede KIPUS. O curso foi implementado a partir do ano 2010 na UFMG e tem permitido o encontro de 61 estudantes, 29 brasileiros e 32 oriundos de outros países do Sul. A análise se baseou no estudo documental e nas respostas a questionários aplicados a egressos do programa e procurou compreender os sentidos de uma pós-graduação de alto nível em educação, com 
um olhar latino-americano. O DLA oferece uma formação gratuita e de qualidade, dando garantias para que os estudantes possam desenvolver seus estudos e interagir com outra língua e cultura e, desde esse viés, aportar novos elementos para a pesquisa em políticas públicas e educação. Os encontros que propicia esse programa têm contribuído com a formação de pesquisadores com um olhar regional amplo e crítico da questão educativa, fortalecendo as parcerias de cooperação e trabalho no tempo. Além disso, essa experiência trouxe para o programa de pós-graduação brasileiro a necessidade de estabelecer relações sólidas com a produção teórico científica latino-americana em educação, levando à formação dos docentes brasileiros para a orientação de teses com perspectivas comparadas na América Latina.

Palavras-chave: Migrações estudiantis Sul Sul, Posgraduação, América Latina, Doctorado Latinoamericano en Educación, UFMG.

\section{Introducción}

Estimular intercambios y migraciones académicas que potencialicen el aprendizaje entre los países del sur, es considerada una política relevante a partir de un proceso que debe fundarse en objetivos y referentes producidos en y para la región. La internacionalización educativa Sur-Sur en los programas de postgrado stricto sensu, constituyen una estrategia importante para enfrentar y superar problemas comunes, en un horizonte de cooperación y solidaridad entre pares. Además, tiene un papel importante para promover la democratización de la educación superior en la región, la construcción de nuevos saberes y la expansión de la innovación endógena. Como lo plantea Gacel-Ávila:

La internacionalización de la educación constituye la respuesta proactiva del mundo académico ante la dinámica global, que favorece una praxis educativa estratégica, proyectada hacia el mundo y coherente con las demandas y necesidades locales para el desarrollo sostenible en un contexto mundializado. Esta dinámica se sustenta en la cooperación, la construcción solidaria de saberes y la transferencia de recursos entre regiones, paises e instituciones. (2018, p. 132).

A pesar de lo anterior, la experiencia de intercambios en América Latina ha sido tradicionalmente dirigida hacia universidades de los países desarrollados tal como se evidencia en los datos sobre movilidad académica, convenios y el carácter de las asociaciones para publicación internacional (Unesco, 2015; OCDE, 2016). Este comportamiento es producto y parte del mantenimiento de una jerarquía entre los sistemas de educación superior alrededor del mundo y al mismo tiempo obstaculiza el crecimiento de la internacionalización Sur-Sur.

En Brasil, la cooperación internacional es señalada como un medio dinamizador del Sistema Nacional de posgraduación, con la captación de conocimiento nuevo y la creación de un ambiente propicio para la innovación, siendo valorizada la intensificación de las relaciones con instituciones del Norte Global, donde está concentrada la mayor parte de las universidades de clase mundial. Por otro lado, el fenómeno de la internacionalización es abordado desde una perspectiva de solidaridad, por la colaboración con otros Estados del Sur, en los cuales la ciencia y la tecnología se encuentran en niveles menos avanzados que en Brasil (Capes, 2010).

Gacel-Ávila, (2018) reporta como en los países latinoamericanos la tasa de intercambio, con relación a la matricula, corresponde apenas al $0.3 \%$ para licenciatura y el $0,03 \%$ en posgrado. Las universidades poseen, mayoritariamente, acuerdos con universidades europeas, americanas y australianas (Castro \& Cabral Neto, 2012). Según Souza (2018) los egresados de las principales universidades de Brasil, México, 
Argentina, Uruguay, Chile, Panamá y Cuba tienen solamente a Cuba como uno de los 10 destinos interesantes para intercambios académicos, sumándose a esta lista Brasil y Chile si se piensa en los 20 países más buscados. A esta tendencia se suman los datos de cooperación internacional para la investigación y publicación de artículos científicos, en las que se mantiene una tendencia hacia los países europeos. En los datos presentados por Gacel-Ávila y Rodríguez-Rodríguez (2017) en la región un 56\% de las instituciones educativas no reporta contar con un programa institucional para apoyar la participación en evento académicos y solo el $65 \%$ presenta estímulos para promover la publicación de artículos científicos.

Una concertación y mayor cooperación en la región no es solo necesaria como importante, pero para que ocurra es necesario, por un lado, la consolidación de los sistemas educativos de la región, pero sobre todo es fundamental la construcción de una identidad colectiva, que establezca lazos de solidaridad y pertenencia. Para Domingues (2009), una integración regional de este tipo solo será posible a través de la construcción de una identidad colectiva para que se conviertan en verdaderos planes de modernización. Esto es lo que se ha intentado, sin mucho éxito, en los casos de los bloques económicos como el Mercado Común del Sur (Mercosur), formado por Argentina, Brasil, Uruguay, Paraguay, Venezuela y Bolivia - este último en proceso de inclusión y Venezuela actualmente suspendido - del que además participan Chile, Colombia, Ecuador, Perú, Guyana y Surinam como asociados; y la Comunidad Andina, formada por Bolivia,
Colombia, Ecuador y Perú. El autor señala que una de las fallas de estos modelos es que no llegan a movilizar profundamente las sociedades que quieren aproximar, todo esto por la falta de una responsabilidad compartida y una identidad común.

Específicamente para educación se encuentran convenios como Andrés Bello ${ }^{1}$, el Sector Educativo del Mercosur ${ }^{2}$, Consejo Suramericano de Educación de la Unión de Naciones Suramericanas ${ }^{3}$ y Alianza del Pacífico ${ }^{4}$. Estos programas funcionan desde una lógica intergubernamental, que envuelve los Ministerios de Educación u otras representaciones oficiales de los países firmantes que acordaron metas y acciones para el desarrollo de la educación superior desde una perspectiva regional. El programa Enlaces $^{5}$ por ejemplo tiene una gestión mixta, con una Comisión de acompañamiento formado por el director del Iesalc $^{6}$, organismo internacional, y por representantes de instituciones de educación superior de diversos países, que cumplen papeles de consultoría, soporte técnico y desarrollo de una agenda de trabajo. Perrota (2016) por su parte, resalta que entre los grupos del sur global el Mercosur es el acuerdo que más avanzo en la institucionalización de políticas para la educación superior en el polo en el que actúa.

Los programas de posgraduación en América Latina han venido cobrando protagonismo y diversificado sus perfiles a lo largo del tiempo. Hoy en día a pesar de la oferta ser mayor, el acceso y la permanencia aún continúan siendo desafíos que hacen que la experiencia de la

\footnotetext{
${ }^{1}$ Tratado firmado en1970, que tiene como objetivo la integración educativa, científica, tecnológica y cultural entre los países da Comunidad Andina.

${ }^{2}$ Espacio de coordinación de las políticas educativas de los países miembros y asociados del Mercosur.

${ }^{3}$ Institución intergubernamental que busca harmonizar los sistemas académicos nacionales de 12 países que lo integran.

${ }^{4}$ Bloque comercial creado en 2012, que en el campo de la educación promueve la movilidad entre los países miembros.

${ }^{5}$ Encuentro Latinoamericano y Caribeño de Educación Superior

${ }^{6}$ Instituto Internacional de la Unesco para la Educación Superior en América Latina y en el Caribe
} 
posgraduación sea muy diferente y desigual en el continente. Políticas con perspectivas neoliberales han posibilitado el establecimiento de programas educativos, que, siendo ofrecidos por universidades privadas o públicas, tienen costos muy altos y porcentajes de duración muy superiores a los inicialmente propuestos. Algunos países han enfocado sus políticas en estrategias de financiación en lugar de oferta, como créditos educativos o, en menor cantidad, con apoyo de entidades no gubernamentales, becas que ayuden al pago de los gastos de colegiatura e investigación.

Estas características sumadas a los contextos sociales y culturales de la región colocan a la oferta educativa y de producción del conocimiento desde el Sur en una desventaja aun mayor de la ya impuesta por el efecto del proceso de colonialidad de poder, del ser y del saber (Escobar, 2000; Lander, 2000; Quijano, 2012) que ha colocado en manos del Norte Global el protagonismo en los discursos y el monopolio del conocimiento. Es por esto por lo que, para contrarrestar esta situación, o al menos desde el escenario educativo, es necesaria la creación de lazos y parcerías en los que la propia región sea el centro de discusión y reconstrucción del saber. Para Gacel-Ávila (2018) el establecimiento de relaciones de hermandad entre las universidades de la región puede nutrir el conocimiento de los países participantes, y con ello aumentar la posibilidad de nuevas fuentes de pares académicos en la investigación y la producción del conocimiento, aprovechando las oportunidades que trae la globalización, la universidad puede ayudar a forjar el futuro de la sociedad.

Las formas de integrar e internacionalizar la educación en la región puede partir de parcerías para investigación, redes de producción de conocimiento, intercambios de estudiantes, profesores e investigadores, pero también puede darse a partir de la creación de programas y sistemas educativos a partir de la concertación y el dialogo entre actores. En este texto presentamos la experiencia del Doctorado
Latinoamericano en educación:

Políticas públicas y Profesión docente (DLA), el proceso de constitución de esta propuesta educativa como parte de un acuerdo entre varios países de América Latina, los objetivos pactados en su establecimiento y la implementación de uno de sus polos en la Universidad Federal de Minas Gerais. Este programa es ejemplo de los alcances que pueden tener procesos educativos construidos a partir de ejercicios de migración e intercambio y a su vez, trae consigo una propuesta interesante que puede inspirar algunos caminos para la posgraduación y la investigación en la región.

\section{El Doctorado Latinoamericano en Educación: Políticas públicas y profesión docente}

El DLA fue construido a partir de un acuerdo firmado entre Universidades de Brasil, Chile, Colombia, Ecuador, Honduras, México, Perú y Venezuela. Con este acuerdo se buscaba la construcción de un programa de postgrado stricto sensu, para promover la formación calificada de doctores en el área educativa, con capacidad para investigar y producir conocimiento sobre la realidad regional. Teniendo un enfoque inicialmente en el estudio de las políticas educativas de la región hoy congrega trabajos que han pensado una gran gama de procesos y sistemas educativos, fortaleciendo así la formación de hacedores de política y de docentes de alta calidad en su práctica y en investigación. Este programa doctoral tiene como marco de fondo la necesidad de posibilitar diálogos y producción de conocimiento educacional de y para la región usando referencias, experiencias y prácticas del contexto como centro del debate.

La propuesta de organizar un doctorado en educación sobre una perspectiva regional fue discutida por primera vez, según la síntesis histórica de Souza (2018), en el ámbito de una reunión en el año 2006 de la Red Kipus - Red de profesores para América Latina y el Caribe. 
Esta es una Red vinculada a la Oficina Regional de Educación para América Latina y el Caribe de la Unesco (Orealc), que congrega instituciones formadoras de profesores de Argentina, Bolivia, Brasil, Chile, Colombia, Costa Rica, Ecuador, Honduras, México, Perú y Venezuela, con el objetivo de contribuir en la discusión sobre políticas públicas nacionales sobre docencia; así como el de apoyar la construcción de alianzas intersectoriales en el fortalecimiento de la profesión docente potencializando el protagonismo de las instituciones formadoras en las transformaciones educativas. Así, en el Encuentro Regional de la Red Kipus, realizado en Venezuela en octubre de 2006, fue firmada una carta de compromiso en la cual instituciones de diferentes países establecían la intención de desenvolver un programa de posgraduación stricto sensu enfocada en la cuestión de la profesión docente, que diera cuenta de las peculiaridades del subcontinente latino americano y caribeño. La discusión fue retomada en agosto de 2007, durante un congreso internacional sobre la evaluación de la función docente realizado en Chile, cuando miembros de la red allí presentes conformaron una nueva agenda para tratar específicamente del doctorado.

La primera reunión de trabajo oficial del DLA se dio en los días 08 y 09 de octubre de 2007, en Venezuela, con el fin de discutir efectivamente la visibilidad de creación del programa, sus propósitos y su sentido. El encuentro fue organizado por la Unesco ${ }^{7}$, a través de la Orealc ${ }^{8}$ y también del Iesalc que había sido vinculado a la iniciativa, con la participación de representantes de las instituciones de la educación superior que se ofrecieron como voluntarios para ser sede en sus países del futuro doctorado - la Universidad Pedagógica Experimental Libertador de Venezuela; la Universidad Pedagógica Nacional de México; la Universidad Pedagógica Nacional de Colombia; la Universidad Tecnológica San Antonio de Márchala de Ecuador; la Universidad Pedagógica Nacional Francisco Morazán de Honduras; la Pontificia Universidad Católica del Perú; la Universidad Federal de Minas Gerais del Brasil; y cuatro instituciones chilenas (Universidad del Bío-Bío, Universidad de la Frontera, Universidad Metropolitana de Ciencias de la Educación y Universidad Católica Cardenal Silva Henríquez). En esa reunión se nombró un grupo técnico ${ }^{9}$ para avanzar en el diseño de la propuesta del DLA, incluyendo el compromiso de los participantes de presentar ante sus instituciones un modelo de Acuerdo de Cooperación Institucional que debería ser firmado por todos. Como resultado, un Acuerdo Internacional fue suscrito por las instituciones de educación superior participantes del encuentro, en el año 2008.

La puesta en marcha de la propuesta original del programa de posgraduación ha tenido diferentes desarrollos en los países vinculados. La Universidad Federal de Minas Gerais fue la primera a institucionalizar el DLA dentro del programa de posgraduación en educación, con abertura de proceso para selección de alumnos en 2009 e inicio en 2010. Este programa, de ingreso anual, en 2020 completa nueve cohortes. En la Universidad Pedagógica de México el DLA se encuentra vinculado al Doctorado de Desarrollo Educativo con Énfasis en Formación de Profesores, a la fecha cuentan con un grupo de alumnos ya graduados y que se encuentran hoy siendo parte del escenario académico mexicano y ecuatoriano, de donde eran oriundos y se espera una nueva llamada para abertura de grupo para

\footnotetext{
${ }^{7}$ Organización de las Naciones Unidas para la Educación, la Ciencia y la Cultura.

${ }^{8}$ Oficina Regional de Educación de la UNESCO para América Latina y el Caribe

${ }^{9}$ Los docentes que participaron de la reunión representando al PPGE/UFMG y que contribuyeron en las articulaciones y concepciones del Doctorado fueron: Dalila Andrade Oliveira, Lucíola Licínio de Castro Paixão Santos, Luiz Alberto Oliveira Gonçalves e Inês Assunção de Castro Teixeira.
} 
El Doctorado Latinoamericano en Educación: una experiencia de internacionalización y migración académica en la posgraduación

el 2021. En la Universidad Pedagógica Experimental Libertador de Venezuela se desarrolló un programa independiente que ha formado ya seis cohortes con excelentes producciones y discusiones para la educación en el país. Mas recientemente, en 2015 en la Universidad Pedagógica Nacional Francisco Morazán de Honduras se inaugura el DLA con un ingreso en el primer año de 40 estudiantes. Ya en las otras universidades el convenio con el DLA se manifiesta hoy como parte de las líneas de trabajo de otros programas ya constituidos en las instituciones, como en el caso de Colombia, donde el DLA se vincula al Doctorado interinstitucional de Educación de Colombia el cual a su vez es ofrecido por tres universidades (Universidad Pedagógica Nacional, Universidad Distrital Francisco José de Caldas y Universidad del Valle).

\section{La experiencia de la Universidad Federal de Minas Gerais y sus alumnos}

En Brasil el DLA se instituye como un curso que integra el Programa de Posgraduación en Educación PPGE/FAE/UFMG desde 2010. El PPGE fue creado en 1971 con el programa de maestría, luego, 20 años después surge el Doctorado. La maestría cuenta hoy con 1.278 trabajos de final de curso aprobados y el doctorado 645 tesis. Estos cursos son ofrecidos en modalidad presencial, formando investigadores en educación, profesores, hacedores de políticas educativas y otros profesionales para diferentes organizaciones sociales y educacionales. Actualmente el programa cuenta con 95 docentes, y más de 400 estudiantes. Y se encuentra organizado en doce líneas de investigación: Educación y Ciencias; Educación y Lenguaje; Sociología de la Educación: Escolarización y Desigualdades Sociales; Educación Matemática; Educación, Cultura, Movimientos Sociales y Acciones Colectivas; Historia de la Educación; Política, Trabajo y Formación Humana; Políticas Públicas de Educación: Concepción, Implementación y Evaluación; Psicología, Psicoanálisis y Educación; Infancia y Educación Infantil;
Docencia: Procesos Constitutivos, Sujetos Socioculturales, Experiencias y Practicas; Currículos, Cultura y Diferencia.

Es en este contexto que se establece el DLA en la universidad minera, convirtiéndose en una de las experiencias más importantes de implementación del acuerdo inicial, constituyéndose también, en la única experiencia de Doctorado Latinoamericano en Educación en Brasil. La UFMG ha sido actor de gran importancia en la construcción de este programa, debido a la capacidad desarrollada a lo largo de su trayectoria. Frente a los otros países firmantes, la UFMG era la que tenía más posibilidades concretas para la implementación de la propuesta, por su estructura física y administrativa, su cuerpo docente de alta calidad, su experiencia en investigación, y por tratarse de una universidad pública gratuita, lo que le daba un atractivo importante para atraer educadores latinoamericanos que en otros contextos no tendrían condiciones para obtener el título de doctor.

El acuerdo inicial entre estos países tenía como visión la construcción de una estructura curricular común de postgrado, y unas condiciones paritarias para la formación de los alumnos. El currículo consiste en 24 créditos, 16 de los cuales son de diciplinas obligatorias, siendo Bases Epistemológicas de la Investigación Educativa (4 créditos); Escenarios y Políticas Públicas en Educación en América Latina (4 créditos); Profesión Docente en América Latina (4 créditos); Seminarios de Investigación y Seminario en Educación (4 créditos); Disciplinas Optativas (6 créditos); Comunicación Científica (2 créditos).

Para los alumnos fueron dispuestas algunas condiciones con el fin de orientar el perfil y el tipo de investigación que se espera sea desarrollado. Los candidatos brasileños, tienen como exigencia que los proyectos de investigación cumplan alguno de los siguientes requisitos: i) tratar de temas educativos, en contraste o comparación de la realidad de países 
latinoamericanos o ii) que estos temas sean, por lo menos, sobre un país de América Latina, excepto el Brasil (UFMG/PPGE, 2013). La movilidad académica representa otra condición obligatoria para los brasileños, que deben permanecer en otro país de la región por un período mínimo de seis meses. De esta manera se promueve efectivamente la formación de doctores brasileños en educación en una perspectiva ampliada, más allá de la nacional. Por su parte para los alumnos extranjeros, el reglamento establece la obligatoriedad de la residencia en la ciudad de Belo Horizonte por lo menos en el primer año. Para este caso se cuenta con una beca otorgada por una agencia de financiamiento nacional, reservada para esta finalidad por el PPGE a cada nueva convocatoria. También se espera que el grupo pueda reunirse en Brasil en el último semestre del doctorado, para la conclusión de la tesis, pero sin garantía de apoyo financiero. En cuanto a la investigación, se espera que ellos realicen trabajos que traten de temas pertinentes a las políticas educativas, al pensamiento pedagógico, a la formación y profesión docente, considerando la diversidad de enfoques teórico-metodológicos y a la realidad de uno o más países de la región.

Además de lo anterior, una política de reciprocidad es establecida con la posibilidad de redirigir las tesis y los trabajos de curso en portugués y en español, así como la oportunidad de cursar español o portugués como segunda lengua dentro de la misma universidad. Los alumnos deben comprobar al final del segundo año el dominio de dos idiomas que en el caso de los hispanohablantes debe incluir el de portugués. Otra característica que llama mucho el interés y garantiza la permanencia de los estudiantes tanto brasileños como extranjeros es la posibilidad de obtener una beca de estudios, por parte de agencias de fomento nacionales a través del programa de posgraduación, para todo el desarrollo de su programa doctoral. Este esfuerzo representa el compromiso de la UFMG con una internacionalización solidaria en el ámbito de América Latina y Caribe. Lamentablemente una estrategia parecida no ha sido garantizada por el PPGE para los brasileños en su proceso de estancia en el exterior, para este propósito, los alumnos han buscado apoyo en algunas entidades de fomento o hasta mismo han corrido con sus propios gastos. Aquellos brasileños con becas nacionales permanecen en sus intercambios con estos mismos recursos, sin otros subsidios.

En la experiencia del DLA de la UFMG el perfil de los estudiantes demuestra algunos datos interesantes que evidencian el comportamiento de la posgraduación en la región, pero que al mismo tiempo pueden ayudar a proyectar el comportamiento de esta, si se piensa desde programas de construcción entre países. Al final del primer semestre de 2020, el DLA acumulaba un total de 61 matrículas desde su primera oferta, siendo 29 de brasileños, 14 colombianos, 6 argentinos, 4 ecuatorianos, 4 peruanos, 2 chilenos, 2 cubanos. Como puede verse en la tabla 1 donde relacionamos las nacionalidades y los años de ingreso. Para esta misma fecha 42 tesis ya habían sido presentadas y las otras 19 se encuentran en desarrollo. La permanencia en el curso y tiempo de aprobación de créditos y de presentación de las tesis es mayoritariamente de 48 meses, con ninguna deserción.

TABLA 1

Número de nuevas matrículas en el doctorado latinoamericano en la UFMG por nacionalidad y por año, 2010-2020

\begin{tabular}{lccccccccccc}
\hline Nacionalidad & \multicolumn{1}{c}{$\mathbf{N}^{\mathbf{0}}$ de nuevas matrículas por año } \\
& $\mathbf{2 0 1 0}$ & $\mathbf{2 0 1 1}$ & $\mathbf{2 0 1 3}$ & $\mathbf{2 0 1 4}$ & $\mathbf{2 0 1 5}$ & $\mathbf{2 0 1 6}$ & $\mathbf{2 0 1 7}$ & $\mathbf{2 0 1 8}$ & $\mathbf{2 0 1 9}$ & $\mathbf{2 0 2 0}$ & Total \\
\hline Brasil & 3 & 2 & 3 & 3 & 5 & 4 & 3 & 4 & 2 & - & 29 \\
Argentina & 1 & 1 & 1 & 1 & 1 & - & 1 & - & - & - & 6
\end{tabular}


El Doctorado Latinoamericano en Educación: una experiencia de internacionalización y migración académica en la posgraduación

\begin{tabular}{lccccccccccc} 
Chile & - & - & 1 & - & - & - & - & 1 & - & - & 2 \\
Colombia & 1 & 1 & 2 & 2 & 1 & 2 & 1 & 2 & 1 & 1 & 14 \\
Ecuador & 1 & 1 & 2 & - & - & - & - & - & - & - & 4 \\
Perú & - & 1 & 1 & - & 1 & - & - & 1 & & - & 4 \\
Cuba & - & - & - & - & - & - & 1 & - & 1 & - & 2 \\
Total & 6 & 6 & 10 & 6 & 8 & 6 & 6 & 8 & 4 & 1 & 61 \\
\hline
\end{tabular}

Nota. Fuente: Elaboración propia con informaciones obtenidas del Programa de Postgrado en Educación de la Universidad Federal de Minas Gerais.

Souza (2018) como parte de su investigación doctoral, titulada "Itinerarios de la Internacionalización de la Educación Superior Brasileña en el ámbito de América Latina y el Caribe", recogió testimonios de exalumnos del doctorado, algunos de los cuales presentamos en este trabajo para ampliar nuestra comprensión sobre la experiencia de este programa. Fueron entrevistados 17 Egresados (10 extranjeros y 7 brasileños) que habían sido alumnos y completado sus tesis en el programa en el periodo 2011 - 2016. Esta investigación conto con todos los protocolos éticos de investigación y se reservan sus nombres como en el trabajo original.

Cuando interrogados por el motivo de no realizar el doctorado en sus países de origen, los extranjeros manifiestan como principales razones el alto costo financiero de la modalidad stricto sensu (3 personas), o por la falta de una oferta de posgraduación y en especial de doctorados en sus países (5 personas). Hubo un sujeto quien señaló que recurrió al Brasil por la calidad del sistema nacional de educación superior y otro que informó que tenía razones laborales, personales y políticas para no continuar los estudios en su país. De los diez encuestados internacionales, tres nunca habían pensado anteriormente en realizar un doctorado en el exterior; otros tres nunca habían pensado en cursarlo en algún otro país vecino; y cuatro ya habían imaginado dar continuidad a su trayectoria académica en un país de otro continente.

La educación comparada es uno de los referenciales de este programa, principalmente por la propia experiencia del programa de pensar la educación en América Latina y hacerlo a partir de las realidades traídas por los propios colegas, y especialmente por la recomendación a los alumnos brasileños de estudiar otra realidad educativa. Sobre esta experiencia los egresados brasileños manifestaron como importante la oportunidad de "profundizarse" en el intercambio, por ser una "experiencia riquísima de investigación y trabajo de campo" (EgresadoDLA_brasileño1 Traducción nuestra) lo que queda evidente en el testimonio de otro de los participantes: "Tuve la oportunidad de descubrir detalles de otro sistema educativo público [...]. Conviví con una organización universitaria diferente de aquella en la que estaba matriculado como doctorando y de aquella en la que soy profesor efectivo. Visite escuelas [de la capital y del interior] del país [donde realice el intercambio]" (EgresadoDLA_brasileño6 Traducción nuestra). En este contexto, enfatizan que el intercambio "favoreció una lectura crítica de la realidad educacional en América Latina", constituyendo una "oportunidad de comprensión del fenómeno educativo de manera más incluyente y plural" (EgresadoDLA_brasileño3 y EgresadoDLA_brasileño5 Traducción nuestra).

Los egresos, nativos y extranjeros, clasificaron como muy relevante la formación propiciada durante la trayectoria en el DLA. Ellos destacaron las contribuciones del punto de vista instrumental, por el perfeccionamiento de las habilidades de producción de conocimiento relativo a la cuestión de las políticas públicas y de profesión docente; las contribuciones desde el punto de vista de las relaciones establecidas, resaltando los lazos de amistad y profesionales que podrán impulsar proyectos colaborativos en el futuro; y, aún los beneficios del punto de vista cultural, una vez que el curso ha inspirado el sentido de pertenencia a América Latina y el 
Caribe. Otro mérito de la movilidad educativa, según los egresados, tiene a ver con la investigación pues esta permite estrechar lazos académicos. Ellos comentan:

por el perfeccionamiento de las habilidades de producción de conocimiento relativo a la cuestión de las políticas públicas y de profesión docente; las contribuciones desde el punto de vista de las relaciones establecidas, resaltando los lazos de amistad y profesionales que podrán impulsar proyectos colaborativos en el futuro; y, aún los beneficios del punto de vista cultural, una vez que el curso ha inspirado el sentido de pertenencia a América Latina y el Caribe. Otro mérito de la movilidad educativa, según los egresados, tiene a ver con la investigación pues esta permite estrechar lazos académicos. Ellos comentan:

La experiencia del sanduiche (nombre dado al intercambio académico) favoreció el establecimiento de intercambios de investigación. Hoy como profesora de educación superior, he buscado establecer proyectos con colegas del país en el que hice mi sanduiche (EgresadoDLA_brasileño4 Traducción nuestra).

Me aproxime a personas que gerenciaban la política pública estudiada en el doctorado (EgresadoDLA_brasileño6 Traducción nuestra).

El DLA presenta un gran potencial para el desarrollo de parcerías internacionales productivas y duraderas. Luego de la conclusión del curso, tres brasileños ya habían regresado al país donde habían hecho el intercambio sea por motivos académicos o profesionales.

Hasta el inicio del año 2020, 42 tesis habían sido presentadas y aprobadas. Estos trabajos presentan una diversidad muy importante entre las temáticas, referenciales y metodologías implementadas, pero todos tienen como común denominador la educación en América Latina. Las tesis aquí producidas son el resultado de la experiencia de un curso que congrega alumnos de diferentes partes de América Latina, que encuentran en el programa un escenario para intercambiar sus experiencias, sus lenguas, sus culturas y la realidad educativa. Los procesos de encuentro, producto de las experiencias individuales y el estudio de las experiencias locales, permiten una visión compleja del contexto educativo latinoamericano.

Para ejemplificar esto, hicimos una revisión bibliográfica de los trabajos producidos por los egresados del programa hasta 2020, y los categorizamos según el objeto de estudio. Aclaramos que esta categorización integra una multiplicidad de abordajes que podrían incluirse subcategorías para explorar con mayor profundidad los aportes que ofrecieron estas tesis doctorales, así como muchos de estos trabajos podrían pertenecer a dos de las categorías propuestas, pero para su definición nos enfocamos en la dirección que los mismos investigadores les dieron a sus trabajos según lo manifestado en los resúmenes de estos. En la tabla 2 puede verse los títulos y autores de los documentos analizados.

\section{Tabla 2}

Producción académica del Doctorado Latinoamericano en Educación: políticas públicas y profesión docente hasta el primer semestre de 2020, por año de ingreso

\begin{tabular}{lcccc}
\hline Estudiante & Nacionalidad & $\begin{array}{l}\text { Director (a) / } \\
\text { Tutor (a) }\end{array}$ & Título del Trabajo \\
\hline & & $\mathbf{2 0 1 0 - \mathbf { 2 0 1 4 }}$ \\
\hline Ana Lúcia Faria & Brasileña & Inês Assunção de & Fora do Quadro: Discursos Sobre
\end{tabular}


El Doctorado Latinoamericano en Educación: una experiencia de internacionalización y migración académica en la posgraduación

de Azevedo

Blanca Olivia Colombiana Pardo Díaz

Breynner Ricardo Brasileño de Oliveira

Maria Elena Ortiz Ecuatoriana Espinoza

Maria José Brasileña

Batista Pinto

Silvia Noemi Argentina

Barco
Castro Teixeira

Regina Helena de Freitas Campos

Maria do Carmo de Lacerda

Peixoto

Marlucy

Paraíso

Alves

Agentes Públicos de Base

Currículos por Competencias en la Educación Infantil: ¿Otras Estrategias de Gobierno de los Infantes?

Ângela Dalben Docência Universitária na Percepção dos Professores de uma Universidade Pública no Brasil e na Argentina no Contexto Atual

Dalila Andrade Políticas de Focalización Educativa Oliveira En Tiempos de Inclusión Educativa. El Caso de Las Escuelas Primarias de Jornada Extendida en la Provincia de Río Negro, República Argentina

\begin{tabular}{|c|c|}
\hline & $2011-2015$ \\
\hline $\begin{array}{l}\text { Ana Maria } \\
\text { Sagrario Tello }\end{array}$ & $\begin{array}{l}\text { Inês Assunção de } \\
\text { Castro Teixeira }\end{array}$ \\
\hline
\end{tabular}

Heslley Machado Brasileño Silva

Eduardo Fleury Mortimer

Trabajo Docente y Políticas
Institucionales en la Universidad Nacional de Rio Cuarto, Argentina, 2011-2015

Professores de Biologia e Ensino de Evolução: uma Perspectiva Comparativa em Países com Contraste de Relação Entre Estado e Igreja na América Latina

Luis Fernando Colombiano Vasquez Zora

André Márcio Políticas Docentes o de Las Picanço Favacho Tecnologías de Formación, Existencia y Desaparición Del Maestro En Colombia

Magaly del Rocio Ecuatoriana

Dalila Andrade

Políticas Educativas y Valorización

Robalino Campos

Oliveira de la Profesión Docente Una Revisión de las Políticas Educativas Nacionales gubernamentales en Ecuador y Perú 1995-2010

Márcio Roberto Brasileño de Lima $\begin{array}{ll}\text { Silvania Sousa do } & \text { Projeto Uca e Plano Ceibal como } \\ \text { Nascimento } & \text { Possibilidades de Reconfiguração da }\end{array}$ 


\begin{tabular}{|c|c|c|c|}
\hline $\begin{array}{l}\text { Ronal Rodolfo } \\
\text { Garnelo Escobar }\end{array}$ & Peruano & $\begin{array}{l}\text { Dalila Andrade } \\
\text { Oliveira }\end{array}$ & $\begin{array}{l}\text { Prática Pedagógica com as } \\
\text { Tecnologias Digitais de Informação } \\
\text { e Comunicação } \\
\text { La Formación Docente Inicial en } \\
\text { el Perú: Cambios y Continuidades } \\
\text { en la Gestión y el Currículo. } \\
(1990-2010)\end{array}$ \\
\hline \multicolumn{4}{|c|}{$2013-2017$} \\
\hline Adenilson Souza & Brasileño & Leôncio & Formação de Professores para a \\
\hline Cunha Júnior & & Gomes Soares & $\begin{array}{l}\text { Educação de Pessoas Jovens e } \\
\text { Adultas no Brasil e no Chile: Um } \\
\text { Estudo Comparado entre o Estado } \\
\text { da Bahia e a Região de Valparaíso }\end{array}$ \\
\hline $\begin{array}{l}\text { Carlos Manuel } \\
\text { Crespo Burgos }\end{array}$ & Ecuatoriano & $\begin{array}{l}\text { Bernardo } \\
\text { Jefferson de } \\
\text { Oliveira }\end{array}$ & $\begin{array}{l}\text { Transformaciones Educativas En } \\
\text { Ecuador y Bolivia (2005 - 2013). } \\
\text { Escenarios En Disputa y Nuevos } \\
\text { Sentidos Par La Educación (Sumak } \\
\text { Kawsay / Suma Qamaña) }\end{array}$ \\
\hline $\begin{array}{l}\text { Daniel Fabian } \\
\text { Roca Flores }\end{array}$ & Peruano & $\begin{array}{l}\text { Rogério Cunha de } \\
\text { Campos }\end{array}$ & $\begin{array}{l}\text { El Trabajo del Profesor en una } \\
\text { Universidad Peruana Originaria de } \\
\text { los Movimientos Estudiantiles }\end{array}$ \\
\hline $\begin{array}{l}\text { Diego Fernando } \\
\text { Bolaños }\end{array}$ & Colombiano & $\begin{array}{l}\text { Marcelo Ricardo } \\
\text { Pereira }\end{array}$ & $\begin{array}{l}\text { 'Respiramos el Mismo Pero Somos } \\
\text { Diferentes' - Constitución de } \\
\text { Subjetividad en Adolescentes } \\
\text { Integrantes de Agrupaciones } \\
\text { Juveniles de Ciudad en Mar del } \\
\text { Plata (Argentina) y Cali (Colombia) }\end{array}$ \\
\hline $\begin{array}{lr}\text { Eugênio } & \text { Magno } \\
\text { Martins } & \text { de } \\
\text { Oliveira } & \end{array}$ & Brasileño & $\begin{array}{l}\text { Inês Assunção de } \\
\text { Castro Teixeira }\end{array}$ & $\begin{array}{l}\text { Fernando Birri e Paulo Freire: } \\
\text { educação e cinema em diálogo como } \\
\text { práticas da liberdade" }\end{array}$ \\
\hline $\begin{array}{l}\text { Felipe Andrés } \\
\text { Zurita Garrido }\end{array}$ & Chileno & $\begin{array}{l}\text { Luciano Mendes } \\
\text { de Faria Filho }\end{array}$ & $\begin{array}{l}\text { Las Políticas } \\
\text { Educacionales sobre el Trablicas } \\
\text { Docente en Chile: Análisis de } \\
\text { la Configuración Estatal de la } \\
\text { Docencia durante la Dictadura } \\
\text { Militar (1973-1990) }\end{array}$ \\
\hline $\begin{array}{l}\text { Gloria Constanza } \\
\text { Aguirre Pinzon }\end{array}$ & Colombiana & $\begin{array}{lr}\text { Ana } & \text { Lydia } \\
\text { Bezerra Santiago }\end{array}$ & 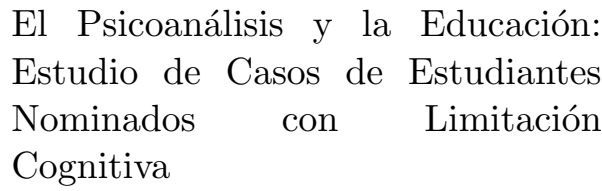 \\
\hline $\begin{array}{l}\text { Leonardo } \\
\text { Marques Soares }\end{array}$ & Brasileño & $\begin{array}{l}\text { Silvania Sousa do } \\
\text { Nascimento }\end{array}$ & $\begin{array}{l}\text { Etnoastronomia, Interculturalidade } \\
\text { e Formação Docente nos Planetários } \\
\text { do Espaço do Conhecimento UFMG } \\
\text { e do Parque Explora }\end{array}$ \\
\hline $\begin{array}{l}\text { María } \\
\text { Villagomez } \\
\text { Rodriguez }\end{array}$ & Ecuatoriana & $\begin{array}{l}\text { Rogério Cunha de } \\
\text { Campos }\end{array}$ & $\begin{array}{l}\text { Práctica de la Interculturalidad, } \\
\text { descolonización y } \text { Formación } \\
\text { Docente. El Programa Académico } \\
\text { Cotopaxi del Ecuador }\end{array}$ \\
\hline
\end{tabular}


El Doctorado Latinoamericano en Educación: una experiencia de internacionalización y migración académica en la posgraduación

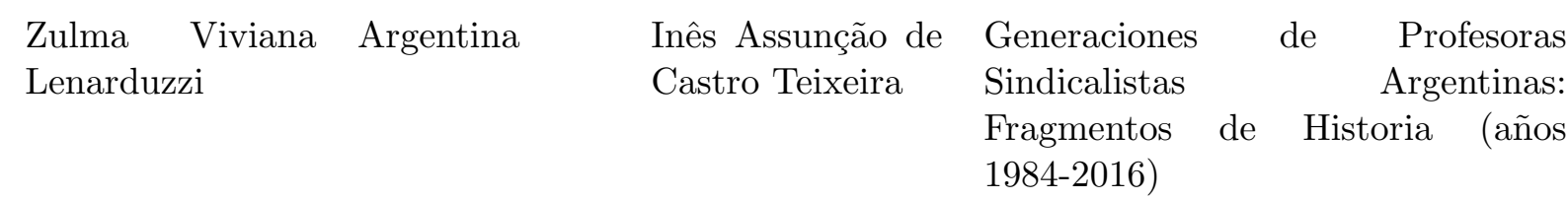

\begin{tabular}{|c|c|c|c|}
\hline \multicolumn{4}{|c|}{ 2014-2018 } \\
\hline Ageu Quintino & Brasileño & Marcus Aurélio & A Política Pública Educacional \\
\hline Mazilão Filho & & $\begin{array}{l}\text { Taborda de } \\
\text { Oliveira }\end{array}$ & $\begin{array}{l}\text { de Simón Rodríguez para Peru e } \\
\text { Bolívia }\end{array}$ \\
\hline $\begin{array}{lr}\text { Denise } & \text { Bianca } \\
\text { Maduro } & \text { Silva } \\
\text { Passades } & \end{array}$ & Brasileña & Rosemary Dore & $\begin{array}{l}\text { Estudo Comparado sobre Evasão } \\
\text { na Educação Profissional - Brasil e } \\
\text { Argentina }\end{array}$ \\
\hline Francisco Javier & Colombiano & Jussara & Contribuciones de ambientes \\
\hline Camelo Bustos & & Araújo & $\begin{array}{l}\text { de modelación matemática a la } \\
\text { constitución de la subjetividad } \\
\text { política }\end{array}$ \\
\hline Fernanda & Colombiana & Arnaldo & Estudio Comparado de las políticas \\
\hline Mejía Toro & & & $\begin{array}{l}\text { Públicas de Educación Ambiental } \\
\text { de Brasil y Colombia }\end{array}$ \\
\hline Miguel & Argentino & Cristina & La institucionalización del saber \\
\hline $\begin{array}{l}\text { Gallegos de San } \\
\text { Vicente }\end{array}$ & & Soares de Gouvea & $\begin{array}{l}\text { psicológico en América Latina } \\
(1900-1940) \text { : un estudio comparado } \\
\text { de sus condiciones intra y extra } \\
\text { disciplinarias }\end{array}$ \\
\hline Oliveira & Brasileña & Francisca Izabel & Programas de Alfabetização de \\
\hline Biondi & & Pereira Maciel & $\begin{array}{l}\text { Pessoas Jovens e Adultas: Brasil e } \\
\text { Argentina uma análise comparada }\end{array}$ \\
\hline & & $2014-2019$ & \\
\hline Eduardo & Bra & Inês Assunção de & Des/Obediência \\
\hline Santos Moura & & Castro Teixeira & $\begin{array}{l}\text { De/Colonialidade da Formação } \\
\text { Docente em Arte na América } \\
\text { Latina (Brasil/Colômbia) }\end{array}$ \\
\hline $\begin{array}{l}\text { Fabrine Leonard } \\
\text { Silva }\end{array}$ & Brasileño & $\begin{array}{l}\text { Luiz Alberto } \\
\text { Oliveira } \\
\text { Gonçalves }\end{array}$ & $\begin{array}{l}\text { Atos infracionais e delitos em } \\
\text { contextos escolares na visão dos } \\
\text { operadores do judiciário em Belo } \\
\text { Horizonte e em Bogotá }\end{array}$ \\
\hline Joaquim Ramos & Brasileño & $\begin{array}{l}\text { Maria de Fátima } \\
\text { Cardoso Gomes }\end{array}$ & $\begin{array}{l}\text { Análise Contrastiva das Estratégias } \\
\text { de Resistência das Crianças Frente } \\
\text { à Regulação dos Adultos em uma } \\
\text { Instituição de Educação Infantil } \\
\text { Brasileira e Duas Colombianas }\end{array}$ \\
\hline $\begin{array}{l}\text { Jorgelina } \\
\text { Tallei }\end{array}$ & Argentina & $\begin{array}{l}\text { Júlio Emílio Diniz } \\
\text { Pereira }\end{array}$ & $\begin{array}{l}\text { La dimensión política e intercultural } \\
\text { en la formación permanente de } \\
\text { docentes que actúan en escuelas } \\
\text { de frontera: análisis del Programa } \\
\text { "pedagogía Intercultural" de la } \\
\text { UNILA }\end{array}$ \\
\hline Juan Arturo & Peruano & Rosemary Dore & Política Educacional no Peru na \\
\hline
\end{tabular}


Maguiña Agüero

Lyda Mayerly Colombiana

González Orjuela

$\begin{array}{lrll}\begin{array}{l}\text { Maria das } \\ \text { Dores Pimentel }\end{array} & \text { Brasileña } & \begin{array}{l}\text { Luiz } \\ \text { de }\end{array} & \begin{array}{l}\text { Alberto } \\ \text { Oliveira } \\ \text { Gogueira }\end{array} \\ \begin{array}{l}\text { Marília } \quad \text { Sousalves/ } \\ \text { Andrade Dias }\end{array} & \text { Brasileña } & \begin{array}{l}\text { Leôncio Soares } \\ \text { Inês Assunção de } \\ \text { Castro Teixeira }\end{array} \\ \begin{array}{l}\text { Gabriel Mancera } \\ \text { Ortiz }\end{array} & \text { Colombiano } & \begin{array}{l}\text { Jussara de Loiola } \\ \text { Araújo }\end{array} \\ \begin{array}{l}\text { Gislene Marino } \\ \text { Costa }\end{array} & \text { Brasileña } & \begin{array}{l}\text { Júlio Emílio Diniz } \\ \text { Pereira }\end{array}\end{array}$

Juliana Santos da Brasileña Conceição

Kildo Adevair dos Brasileña Santos

Miguel Alejandro Colombiano Uribe Martin

Alexandre Fraga Brasileño de Araújo

Castro Teixeira
Transição do Século XX ao Século

XXI: Do Trabalho como Princípio

Educativo ao Empreendedorismo na

Educação

Ana Maria Personas del Conflicto Armado

Rabelo Gomes Colombiano en Situación de

Escolaridad, Una Mirada desde la

Pedagogía Decolonial

A Participação da Universidade e da

Extensão Universitária no Processo

de Colonização do Pensamento na

América Latina

'Para estes meninos e meninas, qual

docência?' (Escolas de Sistemas

Socioeducativos Brasil-Colômbia)

Conocer Reflexivo em Contextos de

Modelación Matemática desde una

Perspectiva Socio Crítica

Formação de Professores de

Música na Argentina: Políticas

Curriculares, Concepções e Modelos

de Formação Docente

Júlio Emílio Diniz Estudo Comparado de Ações de

Pereira Desenvolvimento Profissional de

Professores do Ensino Superior no

Brasil e na Argentina

Dalila Andrade O Pensamento Vivo de José Carlos

Oliveira Mariátegui: Fonte para uma

Pedagogia Latino-Americana

Inês Assunção de Del Tiempo de Docentes entre

Tablero y Pantalla 'Tejidos en la

Experiencia del Tiempo de los

Docentes Bimodales'

Maria Isabel Representações sociais do uso das

Antunes Rocha TICs nas Práticas Pedagógicas

de Educadores dos CEFFAs do

Espírito Santo/Brasil e Santa

Fé/Argentina

Nota. Fuente: Elaboración propia con informaciones obtenidas del Programa de Postgrado en Educación de la Universidad Federal de Minas Gerais. Las tesis se encuentran disponibles en www.repositorios.ufmg.br.

Agrupamos estos trabajos en 6 categorías, aquellos que analizaron o evaluaron Sistemas y/o la experiencia de Programas Educativos específicos, 15 trabajos; trabajos que tienen como enfoque la Formación y el Trabajo Docente, 15 trabajos; Evaluación y Análisis de Políticas Educativas, 7 trabajos; trabajos sobre la Influencia de Políticas Sociales en la Educación, 1 trabajo; trabajos que discursaron sobre Currículos Educativos, 1 trabajo; tesis que analizan la relación de Teorías Psicológicas en la Experiencia Educativa, 3 trabajos. 
El Doctorado Latinoamericano en Educación: una experiencia de internacionalización y migración académica en la posgraduación

La presencia de trabajos mayoritariamente enfocados en analizar la formación y el trabajo docente, así como sistemas y programas educativos, seguido por aquellos que estudian políticas públicas, responde al carácter mismo que constituye el programa doctoral. $\mathrm{Su}$ identidad, así como las disciplinas ofrecidas inclinan la balanza hacía estos intereses, pues tanto la universidad como los alumnos ya traen estas preocupaciones a la hora de escoger el programa. Además, en los primeros años de implementación del programa estas eran las líneas de investigación asociadas. A partir del ingreso de 2014, nuevos profesores y líneas de investigación del programa de posgraduación participan del DLA y con esto se diversifica también los intereses y objetos de investigación. Tanto en los trabajos ya aprobados y publicados como los que están en curso, los objetos de investigación hoy abordan una importante diversidad de temáticas, que honrando los intereses y referentes establecidos en los objetivos del DLA permiten una amplitud de análisis para pensar la educación en América Latina.

\section{Producción académica DLA, según las temáticas}

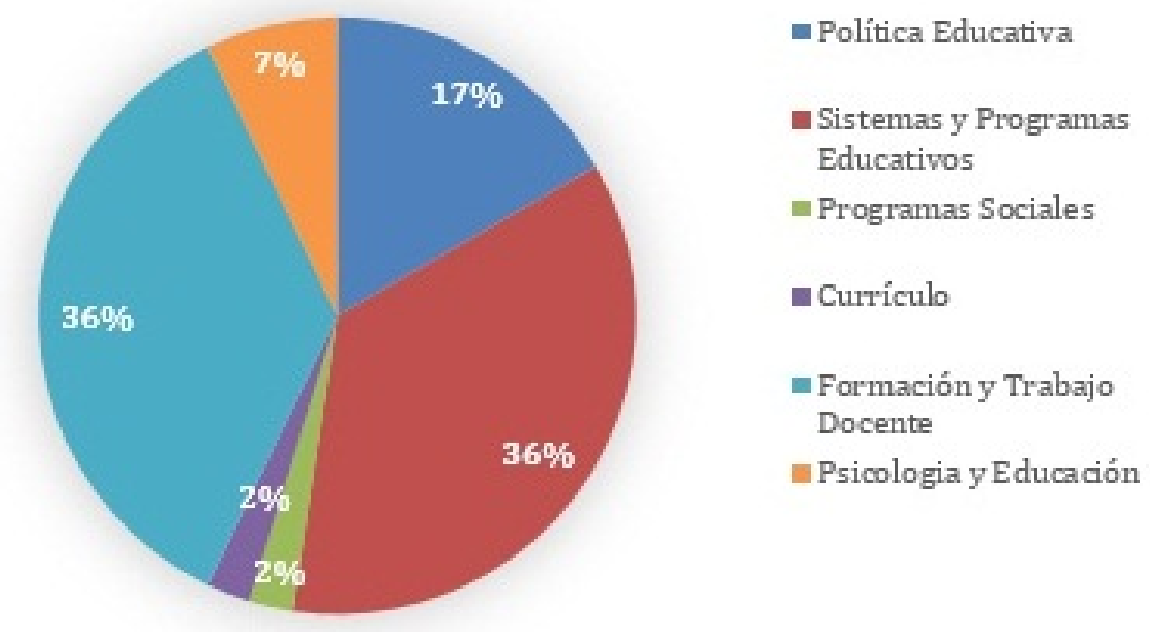

Ilustración 1. Temáticas abordadas en la producción académica del Doctorado Latinoamericano en Educación: Políticas públicas y profesión docente. Elaborado por las autoras.

Los trabajos que tienen como enfoque las políticas públicas y la formación de profesores procuran la interpretación de estrategias y lineamientos que han sido propuestos e implementados en los diferentes contextos que se viven en América Latina. Se destacan particularidades y experiencias, así como se evalúan y se hacen críticas al efecto o impacto que estas han provocado en los lugares donde se han puesto en marcha. Es muy importante que docentes, actores educativos de la región produzcan estudios sobre la formación de docentes y sobre las políticas y sistemas educativos que se generan para la región. Estos estudios pueden servir de referente para la evaluación y elaboración de los lineamientos que orientan la educación en el continente de una forma más crítica y contextualizada del fenómeno estudiado. Tenemos aquí un cuerpo teórico que es referente para pensar cómo se propone la educación en América Latina y cuáles son las posibilidades para repensar la forma de hacer educación y de formar los maestros en y desde el Sur. 
En el trabajo desarrollado por Maduro et al (2019) se presenta la relación de la producción académica del programa con relación a la educación comparada, las autoras destacan como la misma construcción colaborativa, el encuentro de alumnos oriundos de diferentes contextos, las disciplinas ofrecidas y la misma producción académica, se constituyen en una visión crítica de la educación comparada. Los autores defienden como desde este programa se piensa y reflexiona la educación en América Latina desde referentes y experiencias locales, lo que trae un análisis más complejo de las realidades estudiadas e inspira propuestas más contextualizadas. De los trabajos analizados por las autoras, 13 de las tesis aprobadas hasta 2018 presentaban una propuesta de estudio comparado, número que, incluyendo los trabajos de 2019 y 2020 sube a 19 como puede verse en las informaciones presentadas en la tabla dos. Pero se mantiene la tendencia, descrita por las autoras, en cuanto a los países estudiados: experiencias brasileñas relacionadas con otras de países como Argentina, Colombia, México y Uruguay. O trabajos que relacionan experiencias de otros países como Perú y Bolivia, Ecuador y Bolivia, Ecuador y Perú, Argentina y Colombia.

Una de las contribuciones del programa ha sido la importancia dada a explorar el referencial decolonial, como un marco critico que busca integrar autores del Sur a las discusiones de sala de aula y al cuerpo teórico de las tesis. Así, se evidencia la crítica y el interés para integrar otros discursos, referenciales y cosmogonías a la reflexión y producción de conocimiento sobre la educación. Están entre las referencias metodológicas y teóricas de disciplinas y tesis, tanto autores reconocidos por la producción critica en ciencias sociales y educación dentro de la tradición occidental, eurocéntrica, y, al lado de estos, autores del Sur, de diversos orígenes académicos y sociales, referencias directamente relacionadas al movimiento decolonial, así como discursos y críticas desde saberes otros. Este ejercicio fortalece el reconocimiento de los saberes que se producen en esta región y al mismo tiempo permite una visión crítica y contextualizada a la hora de hacer los análisis y escoger los objetos de investigación.

Mas específicamente, el efecto de este esfuerzo puede comprobarse, en la producción de tesis elaboradas desde una perspectiva decolonial (5 trabajos), así como trabajos que han procurado resaltar el trabajo de representantes del pensamiento político pedagógico latinoamericano (3 trabajos) tales como: Mariátegui, Fernando Birri e Paulo Freire, Simón Rodríguez. O la experiencia de programas y sistemas educativos que se fundan desde esta perspectiva (4 trabajos). Además de ser una referencia usada en la crítica y análisis de algunos trabajos que discursan sobre otras realidades educativas (17 trabajos).

El DLA corresponde, en nuestro análisis, un modelo innovador y consistente de internacionalización en el contexto de los programas de postgrado de excelencia. De manera general, el curso ha logrado éxito en el atendimiento a una demanda latente en la región para la titulación en alto nivel de los profesionales en educación. Los egresos, nativos y extranjeros, clasificaron como muy relevante la formación propiciada durante la trayectoria en el DLA. Ellos destacaron las contribuciones del punto de vista instrumental, por el perfeccionamiento de las habilidades de producción de conocimiento relativo a la cuestión de las políticas públicas y de profesión docente; las contribuciones desde el punto de vista de las relaciones establecidas, resaltando los lazos de amistad y profesionales que podrán impulsar proyectos colaborativos en el futuro; y, aún los beneficios del punto de vista cultural, una vez que el curso ha inspirado el sentido de pertenencia a América Latina y el Caribe.

\section{Consideraciones finales}

El DLA ha constituido un espacio privilegiado para discutir y generar conocimiento sobre la América Latina y el Caribe. Este programa ha contribuido, por un lado, para 
afinar el sentido de pertenencia a una cultura regional. Por otro lado, contribuye en la formación de cuadros docentes de alta calidad para países extranjeros, además de fortalecer alianzas que podrán resultar en proyectos y políticas públicas para mejorar la educación. La UFMG al implementar el acuerdo establecido sirve como ejemplo para la región, pues usando su lugar de reconocimiento y posibilidades políticas y económicas, así como del lugar de protagonismo a nivel educativo que tiene en la región promoviendo la colaboración regional generando un lugar de confianza que puede les da la talla a experiencias de otros países fuera de la región. Y se propone a hacerlo pensando en la región, trayendo además de los tradicionales referentes y diálogos desde el Sur y para el Sur.

Se coloca en este trabajo la experiencia de construcción de este proyecto, se resalta la importancia de la elaboración conjunta de programas de posgraduación que respondan a la realidad de la región y que potencialicen el lugar de las universidades y producción de conocimiento desde el Sur. El potencial de este programa radica principalmente en dos de sus características, la posibilidad de construcción conjunta de sus objetivos y propuesta curricular, pero principalmente porque desde sus orígenes y hoy su implementación, se sustenta en el encuentro de académicos, alumnos y profesores migrantes, en intercambios culturales y discursivos. Es allí donde es posible leer, pensar y reflexionar la educación latinoamericana crítica, contextualizada y propositivamente. Las parcerías y productos generados en programas como estos son los que permitirán la consolidación de Latinoamérica como referente y protagonista del discurso del conocimiento y del saber.

Para los estudiantes el DLA es una oportunidad de realizar doctorado en un curso de posgraduación gratuito y de calidad, beneficiarse de becas del gobierno brasileño, conectarse con otra lengua y cultura y aportar elementos nuevos para la investigación en políticas públicas y educación. La socialización en las aulas, en los encuentros entre estudiantes y docentes, la participación en eventos académicos y de movimientos sociales, han contribuido con la formación de investigadores con una visión regional amplia y crítica de la cuestión educativa, fortaleciendo lazos de cooperación y de trabajo duraderos. Además, esta experiencia introdujo en el programa de posgraduación brasileño la necesidad de establecer relaciones sólidas con la producción teórico científica latinoamericana en educación, llevando también la formación de los docentes brasileños a la tarea de orientar tesis desde perspectivas comparada en América Latina.

\section{Referencia}

Capes (2010). Coordenação de Aperfeiçoamento de Pessoal de Nível Superior. Plano Nacional de Pós-Graduação 2011-2020. Brasília: Capes.

Castro, Alda A. y Cabral Neto, Antônio (2012). O ensino superior: a mobilidade estudantil como estratégia de internacionalização na América Latina. Revista Lusófona de Educação. Lisboa: nº 21, p. 69-96.

DLA (2009). Doctorado Latinoamericano en Educación. Programa de Doctorado Latinoamericano en Educación: políticas públicas e profesión docente. Caracas: Documento impreso.

Domingues, José Maurício (2009). A América Latina e a modernidade contemporânea: uma interpretação sociológica. Belo Horizonte: Editora UFMG.

Escobar, Arturo. (2000). El lugar de la naturaleza y la naturaleza del lugar. In: Lander, Edgardo. (org).

La colonialidad del saber: eurocentrismo y ciencias sociales. Perspectivas latinoamericanas, Colección Sur-Sur, CLACSO, Ciudad Autonoma de Buenos Aires, Argentina. 
Gacel-Ávila, Joceline y Rodríguez-Rodríguez, Scilia (2017). Internacionalización de la educación superior en América Latina y el Caribe: un balance. Guadalajara, México: Unesco-Iesalc..

Gacel-Ávila, Joceline (2018). Educación superior, internacionalización e integración en América Latina y el Caribe. In: Henríquez Guajaro, Pedro (Org.). Tendencias de la educación superior en América Latina y el Caribe. Unesco-Iesalc.

Lander, Edgardo. (org). (2000) La colonialidad del saber: eurocentrismo y ciencias sociales. Perspectivas latinoamericanas, Colección Sur-Sur, CLACSO, Ciudad Autónoma de Buenos Aires, Argentina.

Maduro Silva, Denise B., Pintor Flores, Maria José B., e Mejía Toro, Luísa. F. (2019). Otros caminos para la Educación Comparada. Paideia Surcolombiana, (24), 123-139.

https://doi.org/10.25054/01240307.2165

OCDE (2016). Organização para a Cooperação e Desenvolvimento Econômico. Education at a Glance 2016 - OECD Indicators. Paris: OECD Publishing.

Perrota, Daniela (2016). La internacionalización de la universidad: debates globales, acciones regionales. Buenos Aires: Ediciones UNGS.

Quijano, Aníbal. (2012). "Bien vivir": entre el" desarrollo" y la des/colonialidad del poder: entre pensamientos y practicas descoloniales. Revista Viento Sur. v. 1, n. 122

Souza, Juliana de F., Vieira, Lívia M. F. e Toro, Luísa F. M. (2019). Nuestro Norte es el Sur: El Doctorado Latinoamericano en Educación de la UFMG. Educação em Revista, 35, e219688.

https://dx.doi.org/10.1590/0102-4698219688

Souza, Juliana (2018). Itinerários da internacionalização da educação superior brasileira no âmbito da América Latina e Caribe. Tese de doutorado em Educação. Belo Horizonte: UFMG/FaE

UFMG/PPGE (2013). Programa de Pós-graduação em Educação da Universidade Federal de Minas Gerais. Resolução $\mathrm{n}^{\circ} 3$, de 18 de março de 2013. Dá nova redação à Resolução 02/2012 que define normas para o funcionamento do Doutorado Latino Americano. Documento impreso.

UFMG/PPGE (2018). Programa de Pós-graduação em Educação da Universidade Federal de Minas Gerais. Resolução no 14, de 2 de abril de 2018. Dá nova redação à Resolução 03/2013 que define normas para o funcionamento do Doutorado Latino Americano em Educação: Políticas Públicas e Profissão Docente.

Unesco (2015). Organização das Nações Unidas para a Educação, Ciência e Cultura. Unesco Science Report: towards 2030. Paris: Unesco. 


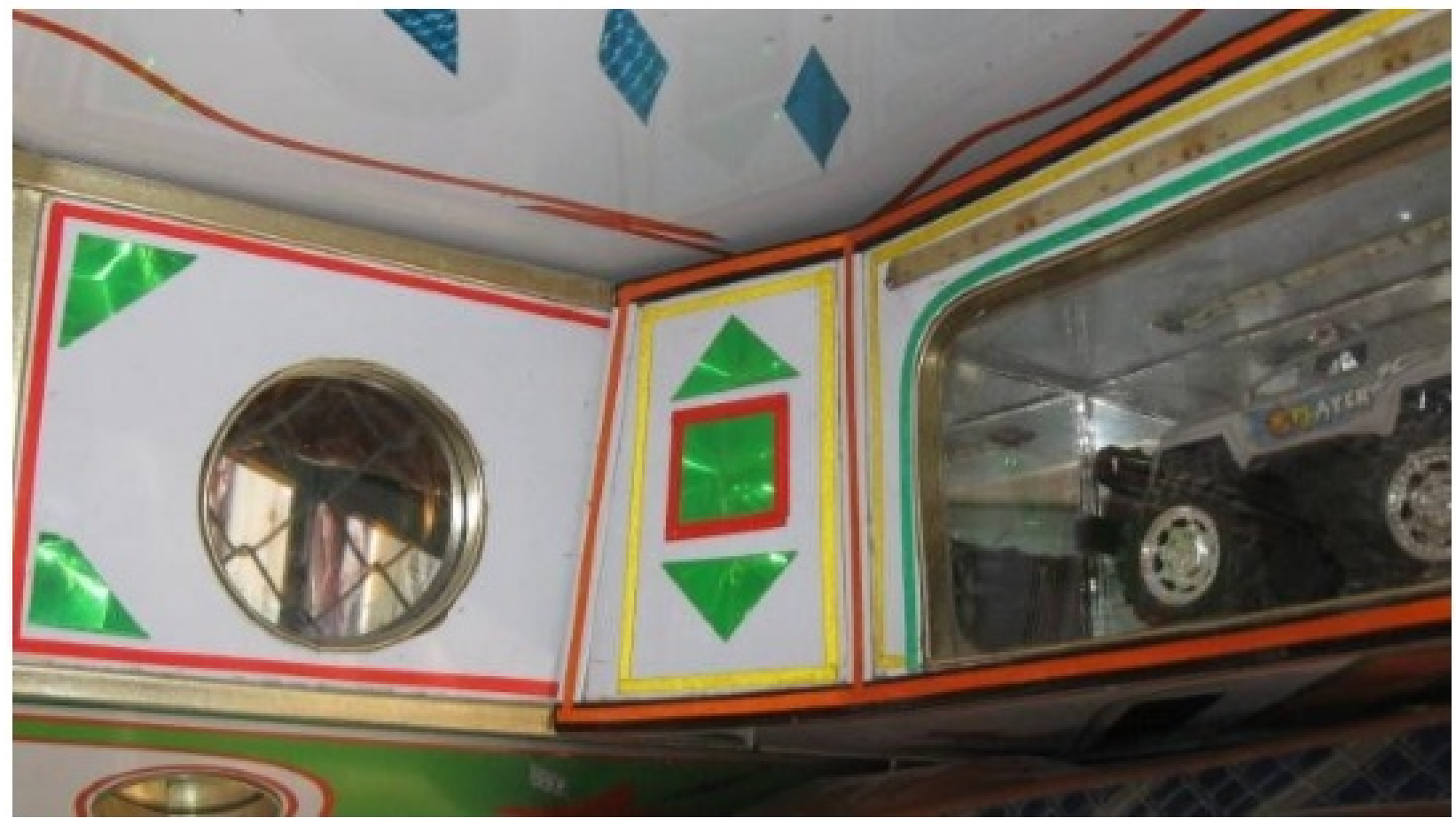

Número de la obra: 85

Título: Chiva "El Lucerito"

Autora: Margarita Chávarro Castro

Lugar: Municipio La Plata, Huila

Fecha de captura: Abril de 2013

Técnica: Fotografía digital

Fuente: Colección Margarita Chávarro Castro 